\title{
Online Hunting and Gathering: An Evolutionary Perspective on Sex Differences in Website Preferences and Navigation
}

\author{
—Eric Stenstrom, Philippe Stenstrom, Gad SaAd, and Soumaya Cheikhrouhou
}

\begin{abstract}
Despite numerous sex differences found in spatial navigation, perception, and verbal abilities, the manner in which these differences manifest themselves in terms of online navigation has yet to be explored. We propose a unified framework based on evolutionary psychology and supported by recent findings in cognitive neuroscience for understanding sex differences in cognition and how they relate to online navigation and website preferences. The literature on sex differences in navigation, object location, spatial rotation, the perception of color, form, and movement, and verbal fluency is reviewed within the context of their evolutionary underpinnings. Based on these findings, specific website design recommendations are proposed. Results of a pilot study examining sex differences in web navigation provide evidence that utilizing an evolutionary approach can engender findings with significant implications for e-communication researchers and practitioners alike.
\end{abstract}

Index Terms-Electronic communication, evolutionary theory, navigation, sex differences, spatial abilities, visual perception, web design.

$\mathbf{R}$ ecent papers have cogently demonstrated that the vestiges of our evolutionary heritage manifest themselves in the digital world. Several researchers have conveyed the importance of examining the evolutionary underpinnings of our cognitive systems and how the evolved design of our communication and foraging mechanisms affect e-communication behaviors [1]-[9]. However, no one has investigated how the sexual division of labor that existed throughout the evolutionary history of humans (i.e., males predominately hunted while females specialized in gathering) [10] has exerted a sex-linked selective pressure on the cognitive mechanisms now involved in e-communication behavior. Despite the multitude of sex differences found in cognitive abilities [11]-[16], only a few studies have examined the differential web browsing preferences of males and females [17], [18], and have done so with minimal theoretical grounding. Practitioners and academics alike would benefit in possessing a theoretical basis for understanding how male and female consumers differentially navigate online environments and respond to various types of web design features.

The main objective of this paper is to extend prior research on Darwinian e-communication by

Manuscript received August 2, 2006; revised June 9, 2007. E. Stenstrom, G. Saad, and S. Cheikhrouhou are with the Marketing Department, John Molson School of Business, Concordia University, Montreal, QC, H3G 1M8, Canada (e-mail: e_stenst@jmsb.concordia.ca; gadsaad@jmsb.concordia.ca; s_cheikh@jmsb.concordia.ca).

P. Stenstrom is with the Department of Psychology, Universite de Montréal, Montreal, QC, H3H 3J7, Canada (e-mail: p.stenstrom@umontreal.ca).

IEEE 10.1109/TPC.2008.2000341 proposing a unified framework for understanding sex differences in online behavior, and putting forth specific website design recommendations to practitioners that are grounded in Darwinian theory and supported by recent findings in cognitive neuroscience. First, the literature on evolutionary psychology and e-communication will be summarized. Next, three main categories of cognitive abilities in which sex differences have been consistently found in the psychology literature will be addressed: (1) spatial ability including spatial navigation, object location, and spatial rotation, (2) perception of color, form, and movement, and (3) verbal fluency. For each category, its evolutionary origins will be discussed, its features in modern men and women as described by the literature will be summarized, and neuropsychological evidence of its biological basis will be briefly examined. The manner and extent to which these evolved sexual dimorphisms in cognition apply to differences in online navigation and website attribute preferences will then be discussed. Finally, the results of a pilot study examining sex differences in online navigation will be reported.

\section{EVOLUTIONARY PSYCHOLOGY AND E-COMMUNICATION}

EvolutionaRY PSYCHOLOGY (EP) is the study of the adaptive functions and phylogenetic history of the mind and how its domain-specific mechanisms have been forged by natural and sexual selection to solve adaptive problems that existed throughout our evolutionary history [19]-[21]. The advantages of this Darwinian approach are its theoretical coherence, its explanatory power in terms of examining the adaptive origins of a particular cognition or 
behavior, as well as its generation of new testable hypotheses that would not have been otherwise posited [22]-[24]. Ned Kock has demonstrated the significance of applying EP to e-communication by putting forth a psychobiological model of e-communication [2]-[5]. The model proposes that the design of the human biological communication apparatus has been forged by natural selection to favor face-to-face, synchronous communication rather than asynchronous and text-intensive forms of e-communication. Specifically, Kock's Media Naturalness Hypothesis makes the case that a greater degree of naturalness of an e-communication medium leads to a decrease in communication ambiguity and cognitive effort, and an increase in physiological arousal. His evolutionary framework explains some contradictory findings within the e-communication literature, particularly as relating to the Media Richness Hypothesis [25].

One aspect of e-communication that has received considerable attention from a Darwinian perspective is online search behavior. Pirolli and Card [7] put forth the INFORMATION FORAGING THEORY of online information seeking based on OPTIMAL FORAGING THEORY (OFT) [26]-[28], which suggests that our cognitive systems have evolved to optimize search efficiency for food and mating opportunities. Pirolli and Card demonstrated that online information seekers use strategies similar to those of food foragers. When searching for information online, browsers tend to visit various websites in search of information much like animals search different patches to find prey. Humans use various information cues referred to as INFORMATION SCENT to estimate the profitability of a source of information in relation to other sources within the online information environment similar to the manner by which animals use the scent of prey to estimate the chances of finding food in various patches. Spink and Cole later proposed to integrate this information foraging model within a single framework of HUMAN INFORMATION BEHAVIOR (HIB), which includes the information seeking, everyday life information seeking, and information use approaches [9], [29].

With regards to shopping behavior, Donald Hantula along with various colleagues proposed a SHOPPING-AS-FORAGING perspective of consumption [1], [8], [30]-[32]. This model examines shopping behavior within a behavioral ecology framework based on OFT that is grounded in evolutionary theory and operant conditioning principles. By examining consumers' behaviors within a "simulated mall," Hantula and his colleagues found that price and feedback delay sensitivity during shopping tasks was consistent with the mathematical predictions of OFT.

In the following sections, we extend the existing evolutionary models of e-communication by examining, within a Darwinian framework, how sex differences in a number of cognitive areas explain and predict sex differences in online behavior.

\section{Sex Differences in Spatial Abilities}

The differential processing of spatial information by the two sexes has received much attention in the psychological and neuropsychological literatures. While researchers consistently find sex differences in a variety of spatial tasks, it is less clear how different spatial abilities relate to each other. For the sake of simplicity we have separated spatial abilities into three categories (spatial navigation, object location, and spatial rotation), each of which possesses its own evolutionary and neuropsychological underpinnings as well as unique implications for the design of online environments.

Spatial Navigation One of the most extensively studied differences between male and female cognition is differential navigational style. It is believed that the differential navigational requirements of HUNTING (i.e., tracking prey across long distances and then finding a direct path home) and GATHERING (i.e., collecting various plants and fruits in close proximity to home base) exerted a sex-linked selective pressure on navigational cognition [15]. Specifically, males are more likely to employ an ORIENTATION (i.e., Euclidean) navigational style because hunting required long-range navigation in expansive novel terrains. Females, however, tend to utilize a LANDMARK (i.e., topographical) navigation style because gathering required short-range navigation in relatively familiar areas close to home base. Ecuyer-Dab and Robert extend this framework by proposing that sex differences in spatial abilities would also have their evolutionary origins rooted in differential mating strategies and survival concerns [33]. They argue that in order to protect their survival and that of their children, females employed a low-risk navigational strategy by staying close to home and focusing on nearby landmarks and spatial cues in order to easily escape from predators and other sources of danger. In contrast, male intrasexual competition for mates required men 
to navigate over extended distances to seek out mating opportunities.

Evidence supports the notion that modern males and females have inherited the predisposition to engage in large-scale and small-scale navigation, respectively. Men provide cardinal references more often than do women when giving directions in relation to a map [34]-[36]. Women, on the other hand, more often supply topological cues such as landmarks, along with the configurational relations between these [34], [35], [37]-[39]. A virtual reality version of the Morris Water Maze task revealed sex differences in the cognitive strategies employed in that women relied predominantly on landmark information whereas men used both landmark and geometric information [40]. Similarly, Lawton found that males and females differ in self-reported wayfinding strategies [41]. Whereas women were more likely to report using a rote strategy by attending to instructions on how to get from place to place, men more often reported using an orientation strategy by maintaining a sense of their own position in relation to environmental reference points. The notion that males make more efficient use of their "internal compass" is further supported by the finding that when being led through virtual mazes, males pointed more accurately at the direction of the maze origin [42]. Also, women tend to report higher levels of anxiety about environmental navigation and make more navigational errors during wayfinding [43]. Past studies have yielded similar sex differences on tasks involving navigation in 2-D environments as compared to those found in naturalistic 3-D environments. Specifically, in tasks involving route following on a 2-D map, 5- to 12-year-old boys make significantly greater use of Euclidean cues, whereas girls of the same age tend to rely more on landmark-based strategies [44], [45]. In sum, males make greater use of their "internal compass" and use both geometric cues and landmarks, whereas women rely predominately on landmarks.

We propose that the aforementioned sex differences in navigational styles translate into differential navigational abilities and preferences in online environments. Our main proposition, as well as the hypotheses tested in the pilot study herewith rest on the premise that the navigation in both online and natural environments utilizes similar cognitive processes. Specifically, we propose that the concepts of wayfinding and landmark usage that apply to spatial navigation through 3-D environments also apply to 2-D-like computer-based interfaces such as websites.
Website navigation can be construed as analogous to spatial navigation in several respects. Websites contain various pages, objects, and landmarks, and are hierarchically structured. In order to navigate through multiple levels of web pages, users must select hyperlinks to "travel" from one page to the next not unlike one's movement from one physical area to another. Males and females have evolved specialized cognitive processes for navigating through space and we propose that more abstract types of navigation -in this case online navigation-use the very same processes. Consistent with this premise is Cox et al.'s finding of sex differences in the programming styles of computer programmers that parallel those found in spatial cognition tasks [46]. Specifically, they examined the manner in which males and females navigate through the abstract space of source code and found that female programmers tended to use a more route-based navigation within the code space as compared to males.

Sex differences in spatial navigation may apply to online navigation in several other distinct ways. Female web navigators may appreciate the use of web elements that act as landmarks by clearly indicating what page and/or section they are on at all times (e.g., a detailed menu bar that is consistently positioned at the top or side of every page). Women may also prefer internet sites that enable navigators to easily return to the homepage via clearly identified hyperlinks found on every page. In addition, a study examining sex differences in website preferences revealed that women preferred to browse through sites with pull-down menus versus those requiring them to click through levels of pages [17]. This finding suggests that women may prefer websites with navigational structures that allow browsing through various sections without leaving the main page, using the pull-down menu as a landmark. Moreover, this finding supports the notion that females rely more on landmark information and less on an internal compass when web browsing. In contrast, males may rely less on landmarks and more on their internal compass to situate themselves while browsing through various levels of depth of a website. In sum, there is evidence that males and females navigate differently in space and that these differences manifest themselves in online environments. In the ensuing two sections we examine how circumstances in our evolutionary past have led to sex differences in other spatial processing abilities, namely object location and spatial rotation. 
Object Location Several studies indicate that women have superior recall of the locations of objects. It has been suggested that this may have stemmed from the greater necessity for ancestral females (as compared to their male counterparts) to remember relative locations of static food sources. Indeed, it is believed that foraging required the rapid learning of the relative positions of plants within diverse vegetations and the recalling of these locations for ensuing seasons [15], [47]. Evidence that such a memory specialization exists in females includes consistent findings that women and young girls outperform men and young boys in tasks measuring memory for object identities and their relative locations in a visual spatial array [15], [47]-[49].

We therefore propose that women will be more adept at learning the locations of products within a wide and dense array of products offered on a single web page of an online store. Moreover, after having previously seen a product of particular interest located within a large number of presented items on a web page, men may find it more difficult to relocate the product for purchase.

Spatial Rotation Although women tend to excel at object location tasks, studies have consistently shown that men perform better than women in tests of mental rotation [16], [50], [51] and more recently in tasks involving the rotation of abstract visual objects presented on computer-based media [52]. It has been suggested that the male advantage in this task evolved from agonistic patterns involving projectiles, namely for hunting [53] as well as navigational activities specific to hunting [54] and mating strategies [33]. The sex-dimorphic nature of spatial rotation processing is readily seen in differential patterns of brain activation between males and females. Functional magnetic resonance imaging (fMRI) studies using mental rotation tasks have revealed sex differences in task-related activation in the temporal, parietal, and perceptual areas [55] as well as frontal areas [56]. In a recent fMRI study, Butler and colleagues found differential patterns of cortical activation during a spatial rotation task that suggest that men utilize an effective, unconscious "bottom-up" neural strategy as opposed to an effortful "top-down" processing for women [57]. This finding suggests that women must expend greater cognitive effort in order to accomplish a 3-D rotation task.

Web page elements requiring mental spatial rotation, such as a series of several photos taken from various angles from which the physical structure of a product is inferred, may therefore be perceived as more meaningful to males as compared to females. In an online shopping task, males perceived the viewing of digital pictures taken from ten different angles as more meaningful than did females [18]. Web designers may consider minimizing mental rotation demands for female users by perhaps offering a full 3-D rendering of a product as opposed to several photographs taken from various angles. However, research is required to investigate whether males and females will respond differently to the 3-D portrayals of products; although women may respond better to $3-\mathrm{D}$ renderings because they minimize the need for cognitive effort involved in mental spatial rotation, men may appreciate the movement of 3-D animations more than women (see the next section).

In sum, the accumulated evidence suggests that important sex differences exist in several key aspects of spatial processing. These differences have clear evolutionary explanations and hold significant implications for the design of online environments (see Table I for a summary of the key findings). In the following section, sex differences in lower-level perception will be examined.

\section{Sex Differences in the Perception of COLOR, FORM, AND MOVEMENT}

Alexander recently proposed that males and females have evolved specialized perceptual systems [58]. This hypothesis explains findings of female superiority in the perception of object form and color as well as a male advantage in the processing of visual motion information, both of which are relevant to the perception of online environments. At the core of Alexander's hypothesis is the well documented finding that the human visual system is segregated into two interrelated [59], [60] but experimentally dissociable [61] processing streams: a PARVOCELLULAR PATHWAY, which deals mainly with form and color processing and is capable of processing information at higher spatial frequencies, and a MAGNOCELLULAR PATHWAY which deals mainly with motion and spatial analysis and is capable of processing information at higher temporal frequencies. Alexander reviews findings indicating that the male and female perceptual systems are biased towards the magnocellular and parvocellular systems, respectively [58]. The author adds that these specialized visual biases arose via natural selection because they led to adaptive sex-dimorphic behaviors. For men, the magnocellular system's specialized processing of 
TABLE I

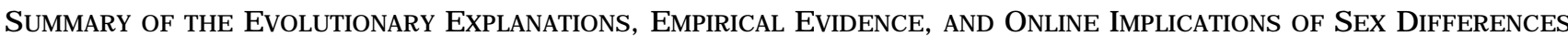
in SPATIAl ABILITIES

\begin{tabular}{|c|c|c|c|c|}
\hline Sex Difference & $\begin{array}{l}\text { Evolutionary } \\
\text { Explanation }\end{array}$ & Empirical Evidence & $\begin{array}{l}\text { Implications for online } \\
\text { environments: evidence }\end{array}$ & $\begin{array}{l}\text { Implications for online environments: } \\
\text { recommendations }\end{array}$ \\
\hline $\begin{array}{l}\text { Navigation: } \\
\text { Females utilize } \\
\text { 'landmark' navigation } \\
\text { style. }\end{array}$ & $\begin{array}{l}\text { Foraging and survival } \\
\text { concerns require } \\
\text { short-range navigation } \\
\text { where home base is } \\
\text { readily found [15], } \\
\text { [33]. }\end{array}$ & $\begin{array}{l}\text { Females supply more } \\
\text { topological cues such as } \\
\text { landmarks and their } \\
\text { configurational relations } \\
\text { [34], [35], [37], [39] and } \\
\text { rely predominantly on } \\
\text { landmark information } \\
\text { [40]. }\end{array}$ & $\begin{array}{l}\text { More females indicated that } \\
\text { websites with pull-down menus } \\
\text { were easier to navigate than } \\
\text { those that require clicking through } \\
\text { levels of pages [17]. } \\
\text { More females reported a } \\
\text { preference for websites that avoid } \\
\text { multiple levels of sub-pages to } \\
\text { navigate through [18]. } \\
\text { Females were less satisfied with } \\
\text { website navigation [18]. }\end{array}$ & $\begin{array}{l}\text { Females will be more likely to prefer (and spend } \\
\text { less time accomplishing tasks in) the wider } \\
\text { navigational structure that allows the navigator } \\
\text { to stay close to the home page. } \\
\text { Females may prefer to have cues that clearly } \\
\text { situate their position in relation to other web } \\
\text { pages/sections (i.e., within the website } \\
\text { structure). } \\
\text { Websites should allow web browsers to stay } \\
\text { close to the home page while navigating via } \\
\text { clearly identified hyperlinks, which enable } \\
\text { immediate return to the home page. } \\
\text { Females may prefer pull-down menus that allow } \\
\text { navigation without leaving the front/main page. }\end{array}$ \\
\hline $\begin{array}{l}\text { Navigation: Males } \\
\text { utilize an orientation } \\
\text { or "internal compass" } \\
\text { navigation style. }\end{array}$ & $\begin{array}{l}\text { Hunting and mating } \\
\text { behaviors required } \\
\text { long-range navigation } \\
\text { in expansive novel } \\
\text { terrain [15], [33]. }\end{array}$ & $\begin{array}{l}\text { When being led through } \\
\text { virtual mazes, males } \\
\text { pointed more accurately } \\
\text { at the direction of the } \\
\text { maze origin [33]. } \\
\text { Males rely on both } \\
\text { geometric and landmark } \\
\text { information [40]. }\end{array}$ & $\begin{array}{l}\text { Males reported spending less } \\
\text { time completing tasks in a } \\
\text { website with a deep as opposed } \\
\text { to a wide navigational layout (see } \\
\text { present pilot study). }\end{array}$ & $\begin{array}{l}\text { Males will be more likely to prefer (and spend } \\
\text { less time accomplishing tasks in) the deeper } \\
\text { navigational structure. } \\
\text { Males may have greater ease in navigating in } \\
\text { online environments with a lesser need for } \\
\text { landmarks/cues, which enable an established } \\
\text { position within the levels of depth of a website. }\end{array}$ \\
\hline $\begin{array}{l}\text { Object Location: } \\
\text { Females' superior } \\
\text { recall of the locations } \\
\text { of objects. }\end{array}$ & $\begin{array}{l}\text { Gathering required } \\
\text { having to rapidly learn } \\
\text { the location of plants } \\
\text { within diverse } \\
\text { vegetations and often } \\
\text { recalling this } \\
\text { information for future } \\
\text { seasons [15]. }\end{array}$ & $\begin{array}{l}\text { Females outperform } \\
\text { males in tasks measuring } \\
\text { memory for object } \\
\text { identities and their } \\
\text { relative locations in a } \\
\text { visual spatial array [15], } \\
\text { [48], [49], [58]. }\end{array}$ & & $\begin{array}{l}\text { After browsing a website, females may possess } \\
\text { the ability to recall a greater number of products } \\
\text { and their locations within the web page. }\end{array}$ \\
\hline $\begin{array}{l}\text { Spatial Rotation: } \\
\text { Males demonstrate } \\
\text { superior spatial } \\
\text { rotation ability. }\end{array}$ & $\begin{array}{l}\text { Hunting required the } \\
\text { accurate pursuit of } \\
\text { and the throwing of } \\
\text { projectiles at moving } \\
\text { prey [15]. }\end{array}$ & $\begin{array}{l}\text { Males perform better } \\
\text { than females on tests of } \\
\text { mental rotation [16], [50], } \\
\text { [52]. }\end{array}$ & $\begin{array}{l}\text { Males found the option of viewing } \\
\text { pictures of a product from various } \\
\text { angles to be more meaningful } \\
\text { when shopping for a digital } \\
\text { camera [18]. }\end{array}$ & $\begin{array}{l}\text { Females may preferably benefit from minimizing } \\
\text { web elements that require mental rotation. }\end{array}$ \\
\hline
\end{tabular}

movement was important for successfully detecting and launching projectiles towards prey and thus critical for hunting. For females, foraging relied on the parvocellular system's sensitivity to object color and form.

The aforementioned sex differences in perceptual systems are supported by a number of empirical studies. For instance, men tend to make more effective use of certain motion-related cues than do females when performing computer-mediated tasks [52]. Also, males prefer to visualize moving objects, which they perceive more vividly than they do static objects [62]. Similarly, one-day-old boys show a greater preference towards moving objects than do one-day-old girls [63]. Furthermore, men exhibit greater accuracy in judging relative velocity of moving objects [64], [65] and generally display more advanced knowledge of the trajectory of moving objects. Superior motion processing translates into a male advantage in the ability to accurately throw projectiles [66], even when controlling for the contributions of height, weight, hand strength, and sport history [67], [68].

Although men appear to have an advantage in motion-related tasks, women tend to surpass men in tasks involving object feature and color processing. For example, girls, compared to boys, show an advantage for object discrimination [69] and color naming [70]. Furthermore, while $8 \%$ of males have inherited color blindness and green-red perception deficiencies, only $0.4 \%$ of females have such disorders [71], [72]. Consistent with the evidence that highlights a female visual bias towards the parvocellular pathway is the finding that the ability to perceive green and red is dependent on precisely this pathway [73]. Thus, the striking genetic sex difference in incidence of color deficiency is explained by considering that dichromat females would be at an enormous survival disadvantage while foraging because of a decreased ability to identify highly caloric foods. Indeed, it has been suggested that the human red-green visual system evolved 
TABLE II

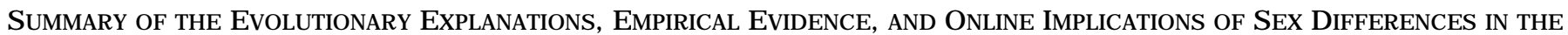
PERCEPTION OF COLOR, FORM, AND MOVEMENT

\begin{tabular}{|c|c|c|c|c|}
\hline Sex Difference & $\begin{array}{l}\text { Evolutionary } \\
\text { Explanation }\end{array}$ & Empirical Evidence & $\begin{array}{l}\text { Implications for Online } \\
\text { Environments: Evidence }\end{array}$ & $\begin{array}{l}\text { Implications for Online } \\
\text { Environments: Recommendations }\end{array}$ \\
\hline $\begin{array}{l}\text { Form: Females' } \\
\text { perceptual bias } \\
\text { towards form. }\end{array}$ & $\begin{array}{l}\text { Foraging required a } \\
\text { specialized parvocellular } \\
\text { visual system for the } \\
\text { visual detection of plants } \\
\text { within diverse } \\
\text { vegetations [58]. }\end{array}$ & $\begin{array}{l}\text { Females show an advantage } \\
\text { for object discrimination [69]. }\end{array}$ & & $\begin{array}{l}\text { Females may possess a greater ability in } \\
\text { discriminating between graphical objects } \\
\text { such as product images, and in learning } \\
\text { the locations of products within wide and } \\
\text { dense arrays on a single web page. }\end{array}$ \\
\hline $\begin{array}{l}\text { Color: Females' } \\
\text { perceptual bias } \\
\text { towards color. }\end{array}$ & $\begin{array}{l}\text { The ability to discriminate } \\
\text { between red and green } \\
\text { had a greater adaptive } \\
\text { value for foragers than } \\
\text { for hunters [58]. } \\
\text { Foraging required a } \\
\text { specialized parvocellular } \\
\text { visual system for } \\
\text { detection of ripe foods } \\
\text { [58]. }\end{array}$ & $\begin{array}{l}\text { Females display an advantage } \\
\text { in color naming [70]. } \\
\text { Females demonstrate a bias } \\
\text { for ventral versus dorsal visual } \\
\text { streams [58]. }\end{array}$ & $\begin{array}{l}\text { In a qualitative analysis, } \\
\text { females reported being more } \\
\text { attracted by a website's colors } \\
\text { [18]. }\end{array}$ & $\begin{array}{l}\text { Females may have a greater appreciation } \\
\text { for the colors used in a website. }\end{array}$ \\
\hline $\begin{array}{l}\text { Color blindness: } \\
8 \% \text { of males have } \\
\text { some degree of color } \\
\text { blindness versus } \\
0.4 \% \text { of females. }\end{array}$ & $\begin{array}{l}\text { For hunting, color } \\
\text { deficiency was } \\
\text { advantageous for } \\
\text { detection of camouflaged } \\
\text { prey [77]. }\end{array}$ & $\begin{array}{l}\text { Dichromats have an enhanced } \\
\text { ability to detect camouflaged } \\
\text { objects [77]. }\end{array}$ & & $\begin{array}{l}\text { When targeting males, underline links, } \\
\text { avoid dark greens or reds within black text, } \\
\text { and avoid using red or green to convey } \\
\text { importance or meaning. }\end{array}$ \\
\hline $\begin{array}{l}\text { Movement: Males } \\
\text { demonstrate a } \\
\text { perceptual bias } \\
\text { towards motion. }\end{array}$ & $\begin{array}{l}\text { Hunting required a } \\
\text { greater attention to and } \\
\text { tracking of moving prey. }\end{array}$ & $\begin{array}{l}\text { Males made more effective } \\
\text { use of certain motion-related } \\
\text { cues in performing computer- } \\
\text { mediated tasks [52]. } \\
\text { One-day-old male infants } \\
\text { display a greater preference } \\
\text { than their one-day-old female } \\
\text { counterparts towards moving } \\
\text { objects [63]. }\end{array}$ & $\begin{array}{l}\text { Males more often reported } \\
\text { preferring websites that make } \\
\text { extensive use of animated } \\
\text { objects and graphics [17]. } \\
\text { Males significantly preferred } \\
\text { the animations and interactivity } \\
\text { of a website [18]. } \\
\text { Males preferred the "flashy" } \\
\text { aspects of a website [18]. }\end{array}$ & $\begin{array}{l}\text { Males may pay more attention to and } \\
\text { make more efficient use of dynamic web } \\
\text { elements. }\end{array}$ \\
\hline
\end{tabular}

to facilitate foraging via the identification of edible red leaves [74] and ripe fruit [75] from green foliage. Furthermore, dichromat males would be advantaged in hunting [76] given that dichromats possess an advantage over trichromats in the detection of color-camouflaged objects [77]. Hence, color-blindness may have endowed some males with an adaptive advantage when hunting by making them better equipped to detect animals, many of which use color camouflage as a predator-avoidance mechanism.

In terms of web design recommendations, as the male perceptual system is more sensitive to moving stimuli, males may pay greater attention to and make more efficient use of dynamic web elements. Consistent with this premise is the finding that a greater number of surveyed males preferred websites that utilized both animated objects and graphics extensively [17]. Similarly, males, as compared to females, preferred the animations and interactivity of a website and more generally its "flashy" aspects [18]. Because dynamic web objects (e.g., animated banner ads) are associated with longer page loading times and higher production costs, designers of websites that are primarily visited by female audiences might consider minimizing the use of animations. Given that women may possess a greater ability to discriminate between graphical objects, they may also respond more favorably to wide and dense arrays of product images. Designers of online environments might also consider sex differences in color scheme preferences. Cyr and Bonanni [18] found that females reported being more attracted by a website's colors than were males. Moreover, one should keep in mind that one in twelve men has a deficiency in perceiving green and/or red. Accordingly, it is recommended that web designers underline links, avoid using dark greens or reds within black text, and minimize the use of red or green when conveying importance or meaning.

In sum, male and female specializations in hunting and gathering, respectively, gave rise to sex-specific biases of the visual system (see Table II for a summary of the key findings). Whereas the male visual system is better suited for the detection of movement, its female counterpart is superior for the processing of color and form. In the following section, the literature on sex differences in verbal abilities will be explored. 
TABLE III

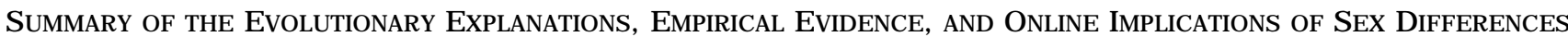
IN VERBAL FLUENCY

\begin{tabular}{|c|c|c|c|c|}
\hline Sex Difference & $\begin{array}{l}\text { Evolutionary } \\
\text { Explanation }\end{array}$ & Empirical Evidence & $\begin{array}{l}\text { Implications for Online } \\
\text { Environments: Evidence }\end{array}$ & $\begin{array}{l}\text { Implications for Online } \\
\text { Environments: Recommendations }\end{array}$ \\
\hline $\begin{array}{l}\text { Verbal Fluency: } \\
\text { Females excel at } \\
\text { verbal fluency tasks. }\end{array}$ & $\begin{array}{l}\text { Hunting requires silence, } \\
\text { whereas foraging and } \\
\text { child rearing activities are } \\
\text { conducive to verbal } \\
\text { communication [11]. }\end{array}$ & $\begin{array}{l}\text { Females outperform men in } \\
\text { numerous tests of verbal ability } \\
\text { [79], [80], particularly verbal } \\
\text { fluency and verbal memory } \\
\text { [48], [81]. }\end{array}$ & & $\begin{array}{l}\text { Males may prefer online textual content } \\
\text { that is presented in a short, concise } \\
\text { manner whereas females may respond } \\
\text { more favorably to websites that have } \\
\text { greater word density and greater lexical } \\
\text { complexity. }\end{array}$ \\
\hline
\end{tabular}

\section{Sex Differences in Verbal Abilities}

Joseph [11] proposed that the sexual division of labor has forged sex differences in verbal ability. During the Plio-Pleistocene, female activities such as child rearing, gathering, and domestic-tool construction promoted verbal communication and led to a female superiority in verbal ability. Verbal communication both with children and fellow gatherers during foraging not only increased productivity but also helped in the caring of offspring and the forging and securing of social networks. It has also been hypothesized that women have used verbal communication more than men throughout human evolution as a form of indirect aggression through gossip in contrast to the more direct physical form that males are more likely to use [78]. Conversely, hunting required males to remain silent while searching for and tracking down prey. While it was likely necessary for males to communicate verbally between hunting excursions in order to strategize, it was critical for hunters (as well as all other predatory animals) to remain as silent as possible during a hunt as the slightest noise could alert the prey and thwart the pursuit [11]. In order to efficiently hunt large game as a group, it is probable that males had to rely on the use of nonverbal communication strategies (e.g., hand gestures) rather than on the use of language.

Several studies have confirmed that women tend to outperform men in tests of verbal ability [79], [80], particularly verbal fluency and verbal memory [51], [81]. Large et al. [82] found that 11- and 12-year-old girls formulated queries in search engines using more words than did boys, and were less likely to perform a one-word query. Furthermore, sex differences in cortical activation patterns during language processing [83] and word generation paradigms [84] suggest that differences in verbal fluency have a neurological basis. Women's superior linguistic abilities may also be associated with a larger CORPUS CALLOSUM, the brain area functionally linking the two cerebral hemispheres. With a more developed bridge between the two hemispheres, the right hemisphere provides women greater assistance during complicated language tasks [85]. Brain imaging studies have supported this notion by demonstrating higher hemispheric lateralization of brain activation patterns in men during language tasks [83], and more specifically when performing complex reading tasks [86], [87].

Based on the above evidence, it is recommended that for males, online textual content should be presented in a succinct and concise manner, whereas women may respond more favorably to websites that have both greater word density and lexical complexity (see Table III for a summary of key findings).

\section{Pilot Study}

An important practical implication of the sex differences discussed in the present paper is that website designers would likely benefit from consideration of the differential navigational styles of their target market. Designing primarily female-oriented, male-oriented, or sex-neutral websites according to the sex ratios of their viewers would contribute to the optimization of browsing efficiency and consumer satisfaction. We present herewith an exploratory pilot study to test the hypothesis that men and women will be differentially effective in product searching through web pages whose layouts are specifically designed to suit their respective evolutionary-based navigational styles.

The manner in which the layout of a website can influence its effectiveness was examined by Vrechopoulos and colleagues who transformed the three most common retail store layouts, namely freeform, grid, and racetrack, into online virtual stores (i.e., website layouts) [88]. The freeform layout has a rather asymmetric and wide structure that enables direct access to all products from the homepage, whereas the racetrack's tunnel-like structure restricts browsers to navigate through 
predetermined paths in order to reach desired products. The researchers found that the grid layout, which provides a hierarchical "tree" structure, was perceived by participants as the easiest and fastest to use. Similarly, Griffith [89] compared the tree structure to a "tunnel" structure analogous to Vrechopoulos and colleagues' racetrack layout and found that participants preferred the tree structure along various metrics (e.g., ease of use, greater elaboration of product information, higher levels of product and brand recall, and greater purchase intention). The literature therefore suggests that individuals clearly prefer a tree structure to other types of web designs. However, the optimal depth and breadth of a tree structure in terms of both the number of levels and of the number of items per level is less apparent. Larson and Czerwinski found that when participants were asked to perform information search tasks in three tree-structured websites $(8 \times 8 \times 8$ [three levels of depth, each with eight items of breadth], $16 \times 32$ [one level with 16 categories and another with 32 items per category], and $32 \times$ 16 [one level with 32 categories and another with 16 items per category]), the most efficient level of breadth and depth in terms of search time was found to be the intermediate design consisting of 16 categories and 32 items per category [90].

In light of the aforementioned sex differences in navigational styles, we hypothesize that males will report spending less time than will females when locating a desired product in a deeper navigational tree structure since males can rely on their "internal compass" as they navigate through various levels of depth. It is also hypothesized that females will report spending less time than will males when locating a desired product in a wider tree structure that allows the navigator to stay close to the homepage without having to browse through numerous levels of depth. This hypothesis rests on the notion that a wider layout better suits the female navigational style as it allows women to stay "close to home" when navigating across the width of the website. The main web page acts as a clear landmark that women can refer back to as they navigate. To reiterate, we posit the following hypotheses:

H1: For the website with a deeper navigational structure, males will report spending significantly less time accomplishing tasks as compared to females.

H2: For the website with a wider navigational structure, females will report spending significantly less time accomplishing tasks as compared to males.

The current study also examined sex differences in disorientation during web navigation.

DISORIENTATION is defined by Ahuja and Webster as "the tendency to lose one's sense of location in a Web site" [91, p.16]. Because disorientation is likely to be caused by losing one's way amongst various levels of navigational depth, we expect disorientation to occur only within the website with the deeper navigational structure. Since the navigational style of men, but not that of women, focuses on an "internal compass," women will report greater perceived disorientation than will men in the deeper website. Specifically, it is proposed that:

H3: Females will report significantly higher perceived disorientation scores than will males for tasks performed within the deep navigational structure.

H4: There will be no significant sex differences in perceived disorientation scores when accomplishing tasks in the website with a wider navigational structure.

\section{Methodology}

The authors created two mock online bookstores for the purposes of the current study (visit www.interdisciplinaryresearch.net/ieee/wide and www.interdisciplinaryresearch.net/ieee/deep to view the websites). The websites were comprised of identical information presented in two different manners. Both websites utilized a tree layout [89], albeit they differed in terms of the number of branches. Both sites featured the same products: 48 book lists, comprised of 10 books each, organized in categories and subcategories, for a total of 480 books. Book lists sequentially presented product information (title, author, price, and a small picture of the book cover) on each of the 10 books on a single page. The "wider" website's homepage contained all categories and subcategories with direct links to the 48 book lists, for a total of two levels of depth. For the "deeper" website, participants were required to navigate through categories and subcategories before reaching the book lists, for a total of five levels of depth. For example, to find a book titled The Third Chimpanzee in the deeper website, the navigational path to reach the book list in which it is situated is: Homepage / Non-Fiction / Academic and Professional / Pure Sciences / Biology. The navigational path to reach the same book list in the 
wider website is simply Biology. The book lists were identical for both websites and no direct link to the homepage was included so that participants had to use the "back" button to return to preceding pages. Websites were matched for visual aesthetics such as color scheme and font.

The data was collected at an Eastern Canadian city using snowball sampling. It was gathered using an internet-based survey and a self-administered questionnaire sent by email. Respondents were asked to complete two task sets, one on each website. For each task, they were given a set of three book titles and were instructed to locate each book. Once a book was found, respondents were to write its posted prices in the questionnaire. Prior to the book-finding tasks, participants were instructed to track and record the time in minutes and seconds spent completing each task set. This recorded time was used as the measure of navigational effectiveness. Participants were then asked to respond to questions measuring the level of perceived disorientation felt while performing the book-finding tasks, which consisted of a seven-item scale developed and validated by Ahuja and Webster [91]. Items include the following: I felt disoriented, It was difficult to find a page that I had previously viewed, and I didn't know how to get to my desired location. The final section of the questionnaire consisted of measures of web skill, prior knowledge of the product (books), web usage, internet connection speed, and demographics (sex and age). Internet expertise was measured using two items adapted from Novak, Hoffman, and Yung [92]: I am skilled at using the web and I know somewhat more than most users about using the $w e b$. Prior knowledge of the product was assessed with one item: I consider myself knowledgeable about various book categories and titles. Two items were used to evaluate internet usage: How many hours per week do you use the internet? and How many hours per week do you use the internet for shopping?

The orders of both the websites and the tasks were counterbalanced, yielding a 2 (task order) $\times 2$ (website order) within-subjects design. Participants were randomly assigned to each treatment condition. All conditions were matched for sex. Post hoc analyses revealed a learning effect whereby experience on the first website affected performance on the second website differently for the two sexes. The exploration of this asymmetrical learning effect is beyond the scope of the current article but will likely be addressed in future research. Thus, all analyses were undertaken solely on the items related to the first website browsed, following a 2 $\times 2$ between-subjects design. Of the 67 distributed questionnaires, 35 completed questionnaires were received. One questionnaire was discarded due to missing values yielding 34 usable questionnaires. The sample consisted of 15 males and 19 females. Because the assumption of normality was violated (likely due to the small sample size), Mann-Whitney nonparametric tests were used in all analyses.

\section{RESULTS}

The descriptive profile of this sample yielded neither significant sex differences for demographics and experience variables ( $p$-values ranging from 0.40 to 0.99 ), nor for internet connection speed (Pearson Chi-square $=2.63 ; d f=2 ; p=0.27$ ). In addition, all reported book prices were accurate, demonstrating that all tasks were completed properly.

As hypothesized $(\mathrm{H} 1)$, males $(N=9$, Median $[\mathrm{MD}]=$ 3.53 , Inter-quartile range $[\mathrm{IQR}]=3.11$ ) reported spending significantly less time completing the tasks within the deeper website than did females $(N=9, \mathrm{MD}=7, \mathrm{IQR}=2.52 ; p=0.01)$. However, contrary to $\mathrm{H} 2$, there were no significant sex differences in the time reported to have been spent accomplishing tasks in the wider website between males $(N=6, \mathrm{MD}=8, \mathrm{IQR}=6.65)$ and females $(N=10, \mathrm{MD}=7.99, \mathrm{IQR}=5.75 ; p=0.79)$. Males reported spending significantly less time completing the tasks in the deeper website $(N=9, \mathrm{MD}=3.53$, $\mathrm{IQR}=3.11)$ than in the wider website $(N=6$, $\mathrm{MD}=8, \mathrm{IQR}=6.65 ; p=0.068)$. However, there were no significant differences in the reported times between the deeper $(N=9, \mathrm{MD}=7, \mathrm{IQR}=2.52$ ) and the wider $(N=10, \mathrm{MD}=7.99, \mathrm{IQR}=5.75$; $p=0.838$ ) websites for females. As illustrated in Fig. 1, males reported accomplishing the task in the wider website at a similar speed to females in both the deeper and the wider websites, whereas males reported spending significantly less time accomplishing tasks when navigating through the deeper website.

The disorientation items yielded a Cronbach alpha of 0.88 , thus reaffirming the scale's reliability. Item-to-total correlations did not show any potentially significant improvement if any scale item were to be discarded. Thus, disorientation scores were averaged using the seven items. No significant sex differences in disorientation scores were found, irrespective of the website browsed. Females $(N=9, \mathrm{MD}=2.86, \mathrm{IQR}=3.07)$ did not report significantly higher disorientation scores 


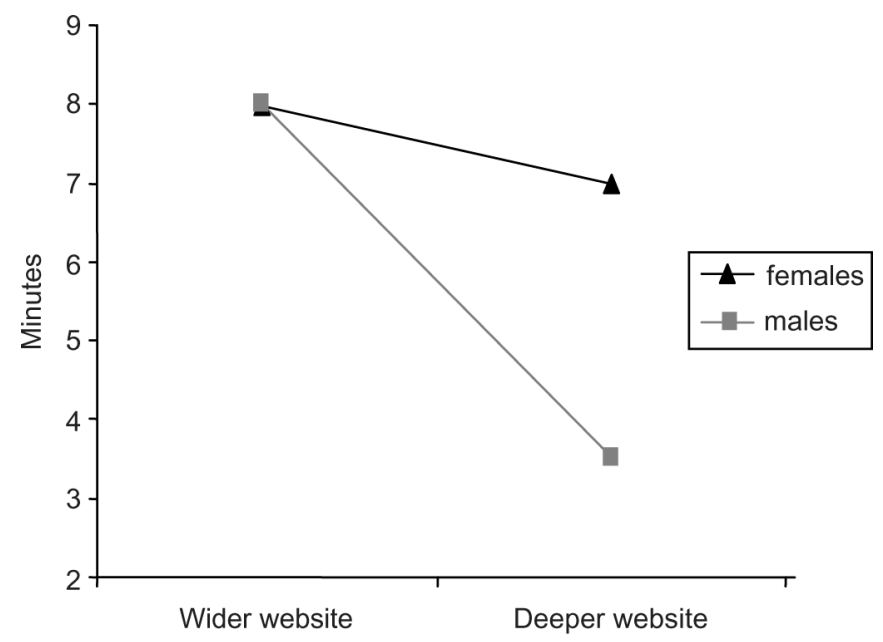

Fig. 1. Sex differences in time spent accomplishing the task set.

than did males when accomplishing tasks within the deeper website $(N=9, \mathrm{MD}=3.57, \mathrm{IQR}=2.72$; $p=0.627$ ), thus refuting H3. There were no significant sex differences in the disorientation scores in the wider website (males: $N=6$, $\mathrm{MD}=3.93, \mathrm{IQR}=3.18$; females: $N=10, \mathrm{MD}=4.21$, $\mathrm{IQR}=2.25 ; p=0.828$ ), as postulated in $\mathrm{H} 4$. No significant within-sex differences in disorientation scores were found when comparing the wide and deep sites (males: $p=0.289$; females: $p=0.236$ ).

\section{Discussion}

As hypothesized, men reported spending significantly less time completing tasks in the deeper website than did women (H1). Moreover, men reported spending significantly less time completing tasks in the deeper structure than in the wider layout. That this increase in reported effectiveness was not seen in women further indicates that the navigational structure of the deep website layout was better suited for the male navigational style. This finding is consistent with the notion that males, as compared to females, make greater use of their "internal compass" when navigating through multiple levels of a website. The male advantage in the deep website layout was unrelated to sex differences in terms of internet skill and usage, computer expertise, or prior knowledge of the product. However, women reported spending as much time as did men in completing tasks within the wider website (H2) and reported taking equal time for both websites. The results suggest that for women, product search effectiveness may not be affected by the manipulation of levels within a website, whereas a deeper navigational structure may benefit males.

Although the data support the hypothesis that no sex differences would be found for perceived disorientation measures in the wide website (H4), the hypothesis that such differences would be found in the deep website was not supported (H3). The finding that women reported no differences in perceived disorientation across websites may parallel the fact that women reported performing equally well on both sites.

It is conceivable that no significant differences were found in the reported times and disorientation scores between websites for females due to the inherent simplicity of both the websites and experimental tasks. Future research utilizing more complex website structures, larger sample sizes, and random sampling methods are likely needed. The current study's use of self-reported time is an additional limitation given that the accuracy of the time measurements cannot be verified. Future studies examining navigational effectiveness would likely benefit from incorporating more objective measures of time such as experimenter-controlled or software-mediated measurements in conjunction with self-reported time. Tracking precise search paths (e.g., using "clickstream" technology) might also yield interesting findings. Overall, the results demonstrate that the manipulation of website structure-in this case, the number of navigational levels - is likely to affect males and females differentially. This finding suggests that sex differences in online behavior can be addressed through the manipulation of online environments.

\section{ConCLusion}

Sex appears to be an important variable in models of e-communication. Sex differences are found at numerous levels of human cognition, from LOWER-LEVEL PERCEPTION (detection of movement and object features) to HIGHER-ORDER COGNITION (language processing). Whereas recent advances in the cognitive neurosciences have uncovered a biological basis for many of these differences, evolutionary psychology provides a consilient framework from which these findings can be integrated and understood. It should be noted that this evolutionary approach does not preclude the role of socialization and idiosyncractic life experiences [93] in the development of the sex differences reported in this paper. However, the body of evidence supporting a significant biological component is compelling, particularly 
when considering that sex differences in cognitive abilities have been found in young children (i.e., before substantial differences in life experiences might arise) and across various cultures (see [13] for review). It is proposed that practitioners can increase the functionality and popularity of their websites by considering these sex differences from a Darwinian perspective.

Practitioners would likely benefit from designing websites in a manner that optimizes navigational efficiency and consumer satisfaction in accordance with the sex ratio of their target market. Web designers should consider tailoring the navigational structure, layout, color scheme, graphics, dynamic web elements, 3-D representations of products, and verbal complexity of websites accordingly. In addition, practitioners targeting audiences with a near-equal sex ratio may consider building one website for each sex and subsequently using cookies to automatically redirect the consumers to the suitable website [17]. While the current paper focuses on online environments, many of the aforementioned concepts are applicable to an understanding of sex differences in other forms of computer-based interfaces and related digital media. For example, the fact that males comprise the large majority of the video game market [94] may be explained in part by the male navigational style and preference for moving objects and interactive media. The design recommendations put forth in this paper can be empirically tested in future research, and sex differences in preferences for other forms of media such as advertising could also be investigated.

A Darwinian perspective may shed light as to how certain features of digital media are differentially appreciated by each sex. With millions of individuals accessing the internet on a daily basis for health, financial, and government information, as well as shopping, entertainment, and job searching [95], practitioners cannot afford to assume that both sexes perceive and appreciate this digital realm in identical ways.

\section{ACKNOWLEDGMENT}

The authors wish to thank D. Bélisle for assistance in data scoring and analyses, C. Solomon for editorial assistance, and R. Stenstrom for statistical consultation.

\section{REFERENCES}

[1] D. A. Hantula, D. F. DiClemente, and A. K. Rajala, "Outside the box: The analysis of consumer behaviour," in Organizational Change, L. Hayes, J. Austin, R. Houmanfar, and M. Clayton, Eds. Reno, NV: Context Press, 2001, pp. 203-223.

[2] N. Kock, "The ape that used email: Understanding e-communication behavior through evolution theory," Commun. AIS, vol. 5, no. 3, pp. 1-29, 2001.

[3] N. Kock, "Compensatory adaptation to a lean medium: An action research investigation of electronic communication in process improvement groups," IEEE Trans. Prof. Commun., vol. 44, no. 4, pp. 267-285, Dec., 2001.

[4] N. Kock, "The psychobiological model: Towards a new theory of computer-mediated communication based on Darwinian evolution," Organization Sci., vol. 15, no. 3, pp. 327-348, 2004.

[5] N. Kock, "Media richness or media naturalness? The evolution of our biological communication apparatus and its influence on our behavior toward e-communication tools," IEEE Trans. Prof. Commun., vol. 48, no. 2, pp. 117-130, Jun., 2005.

[6] N. Kock and D. A. Hantula, "Do we have e-collaboration genes?," Int. J. e-Collaboration, vol. 1, no. 2, pp. i-ix, 2005.

[7] P. L. Pirolli and S. K. Card, "Information foraging," Psychol. Rev., vol. 106, no. 4, pp. 643-675, 1999.

[8] A. K. Rajala and D. A. Hantula, "Towards a behavioral ecology of consumption: Delay reduction effects on foraging in a simulated online mall," Managerial Decision Econ., vol. 21, no. 3/4, pp. 145-158, 2000.

[9] A. Spink and C. B. Cole, "Human information behavior: Integrating diverse approaches and information use," J. Amer. Soc. Inform. Sci. Technol., vol. 57, no. 1, pp. 25-35, 2006.

[10] J. Tooby and I. DeVore, "The reconstruction of hominid behavioral evolution through strategic modeling," in The Evolution of Human Behavior: Primate Models, W. G. Kinzey, Ed. Albany, NY: SUNY Press, 1987, pp. 183-239.

[11] R. Joseph, "The evolution of sex differences in language, sexuality, and visual-spatial skills," Archives of Sexual Behaviour, vol. 29, no. 1, pp. 35-66, 2000.

[12] D. Kimura, Sex and Cognition. Cambridge, MA: MIT Press, 1999.

[13] D. Kimura, "Sex hormones influence human cognitive pattern," Neuroendocrinology Lett., vol. 23, no. 4, pp. 67-77, 2002.

[14] E. A. Maguire, N. Burgess, and J. O'Keefe, "Human spatial navigation: Cognitive maps, sexual dimorphism, and neural substrates," Current Opinion Neurobiol., vol. 9, no. 2, pp. 171-177, 1999.

[15] I. Silverman and M. Eals, "Sex differences in spatial abilities: Evolutionary theory and data," in The Adapted Mind: Evolutionary Psychology and the Generation of Culture, J. H. Barkow, L. Cosmides, and J. Tooby, Eds. New York: Oxford Univ. Press, 1992, pp. 533-549.

[16] D. Voyer, S. Voyer, and M. P. Bryden, "Magnitude of sex differences in spatial abilities: A meta-analysis and consideration of critical variables," Psychol. Bull., vol. 117, no. 2, pp. 250-270, 1995. 
[17] S. J. Simon, "The impact of culture and gender on web sites: An empirical study," The Data Base for Advances in Information Systems, vol. 32, no. 1 , pp. 18-37, 2001.

[18] D. Cyr and C. Bonanni, "Gender and website design in e-business," Int. J. Electron. Bus., vol. 3, no. 6, pp. 565-582, 2005.

[19] D. M. Buss, Evolutionary Psychology: The New Science of the Mind. Boston, MA: Allyn and Bacon, 1999.

[20] S. Pinker, The Blank Slate: The Modern Denial of Human Nature. New York: Penguin Putnam, 2002.

[21] J. Tooby and L. Cosmides, "The psychological foundations of culture," in The Adapted Mind: Evolutionary Psychology and the Generation of Culture, J. H. Barkow, L. Cosmides, and J. Tooby, Eds. New York: Oxford Univ. Press, 1992, pp. 19-136.

[22] G. Saad, “Applying evolutionary psychology in understanding the Darwinian roots of consumption phenomena," Managerial Decision Econ., vol. 27, no. 2-3, pp. 189-201, 2006.

[23] G. Saad, The Evolutionary Bases of Consumption. Mahwah, NJ: Lawrence Erlbaum Assoc., 2007.

[24] G. Saad and T. Gill, "Applications of evolutionary psychology in marketing," Psychol. Marketing, vol. 17, no. 12, pp. 1005-1034, 2000.

[25] R. L. Daft and R. H. Lengel, "Organizational information requirements, media richness and structural design," Manage. Sci., vol. 32, no. 5, pp. 554-571, 1986.

[26] J. M. Emlen, "The role of time and energy in food preference," Amer. Naturalist, vol. 100, no. 916, pp. 611-617, 1966.

[27] R. H. MacArthur and E. R. Pianka, "On the optimal use of a patchy environment," Amer. Naturalist, vol. 100, no. 916, pp. 603-609, 1966.

[28] D. W. Stephens and J. R. Krebs, Foraging Theory. Princeton, NJ: Princeton Univ. Press, 1986.

[29] A. Spink and C. Cole, "A human information behavior approach to the philosophy of information," Library Trends, vol. 52, no. 3, pp. 373-380, 2004.

[30] C. Smith and D. A. Hantula, "Pricing effects on foraging in a simulated internet shopping mall," $J$. Econ. Psychol., vol. 24, no. 5, pp. 653-674, 2003.

[31] D. DiClemente and D. A. Hantula, "Time sensitivity in online shopping: Extensions of the foraging model," Psychol. Marketing, vol. 20, no. 9, pp. 785-809, 2003.

[32] D. DiClemente and D. A. Hantula, "Applied behavioral economics and consumer choice," $J$. Econ. Psychol., vol. 24, no. 5, pp. 589-602, 2003.

[33] I. Ecuyer-Dab and M. Robert, "Have sex differences in spatial ability evolved from male competition for mating and female concern for survival?,” Cognition, vol. 91, no. 3, pp. 221-257, 2004.

[34] J. M. Dabbs, E. L. Chang, R. A. Strong, and R. Milun, "Spatial ability, navigation strategy, and geographic knowledge among men and women," Evolution and Human Behavior, vol. 19, no. 2, pp. 89-98, 1998.
[35] D. R. Montello, K. L. Lovelace, R. G. Golledge, and C. M. Self, "Sex-related differences and similarities in geographic and environmental spatial abilities," Ann. Assoc. Amer. Geographers, vol. 89, no. 3, pp. 515-534, 1999.

[36] S. L. Ward, N. Newcombe, and W. F. Overton, "Turn left at the church, or three miles north: A study of direction giving and sex differences," Environment and Behavior, vol. 18, no. 2, pp. 192-213, 1986.

[37] J. Choi and I. Silverman, "Sexual dimorphism in spatial behaviors: Applications to route-learning," Evolution and Cognition, vol. 2, no. 2, pp. 165-171, 1996.

[38] L. A. M. Galea and D. Kimura, "Sex differences in route learning," Personality and Individual Differences, vol. 14, no. 1, pp. 53-65, 1993.

[39] L. K. Miller and V. Santoni, "Sex differences in spatial abilities: Strategic and experiential correlates," Acta Psychologica, vol. 62, no. 3, pp. 225-235, 1986.

[40] N. J. Sandstrom, J. Kaufman, and S. A. Huettel, "Males and females use different distal cues in a virtual environment navigation task," Cognitive Brain Res., vol. 6, no. 4, pp. 351-360, 1998.

[41] C. A. Lawton, "Gender differences in way-finding strategies: Relationship to spatial ability and spatial anxiety," Sex Roles, vol. 30, no. 11/12, pp. 765-779, 1994.

[42] C. A. Lawton and K. A. Morrin, "Gender differences in pointing accuracy in computer-simulated 3D mazes," Sex Roles, vol. 40, no. 1-2, pp. 73-92, 1999.

[43] H. Akio and N. Yoshiaki, "Sex differences in wayfinding behavior using well- or poorly-written route descriptions," Tohoku Psychologica Folia, vol. 63, pp. 15-24, 2004.

[44] A. C. Gibbs and J. F. Wilson, "Sex differences in route learning by children," Perceptual and Motor Skills, vol. 88, no. 2, pp. 590-594, 1999.

[45] J. L. Kersker, M. L. Epley, and J. F. Wilson, "Sex differences in landmark learning by children aged 5 to 12 years," Perceptual and Motor Skills, vol. 96 no. 1, pp. 329-338, 2003.

[46] A. Cox, L. Zhao, and M. Fisher, "Sex differences in abstract spatial cognition," presented at the Annual Meeting of the Human Behavior and Evolution Soc., Philadelphia, PA, Jun., 7-1 1, 2006.

[47] G. M. Alexander, M. G. Packard, and B. S. Peterson, "Sex and spatial position effects on object location memory following intentional learning of object identities," Neuropsychologia, vol. 40, no. 8, pp. 1516-1522, 2002.

[48] M. Eals and I. Silverman, "The hunter-gatherer theory of spatial sex differences: Proximate factors mediating the female advantage in recall of object arrays," Ethology and Sociobiol., vol. 15, no. 2, pp. 95-105, 1994.

[49] L. J. Levy, R. S. Astur, and K. M. Frick, "Men and women differ in object memory but not performance of a virtual radial maze," Behavioral Neurosci., vol. 199, no. 4, pp. 853-862, 2005.

[50] R. N. Shepard and J. Metzler, "Mental rotation of three-dimensional objects," Science, vol. 171, no. 972, pp. 701-703, 1971. 
[51] D. Kimura, "Sex, sexual orientation and sex hormones influence human cognitive function," Current Opinion Neurobiol., vol. 6, no. 2, pp. 259-263, 1996.

[52] G. S. Hubona and G. W. Shirah, "The gender factor performing visualization tasks on computer media," in Proc. 37th Annu. Hawaii Int. Conf. System Sciences, 2004, p. 40097.3.

[53] D. Kolakowsky and R. M. Malina, "Spatial ability, throwing accuracy, and man's hunting heritage," Nature, vol. 251, no. 5474, pp. 410-412, 1974.

[54] D. C. Geary, "Sexual selection and sex differences in spatial cognition," Learning and Individual Differences, vol. 7, no. 4, pp. 289-301, 1995.

[55] K. Jordan, T. Wustenberg, H.-J. Heinze, M. Peters, and L. Jancke, "Women and men exhibit different cortical activation patterns during mental rotation tasks," Neuropsychologia, vol. 40, no. 13, pp. 2397-2408, 2002.

[56] E. Weiss, C. M. Siedentopf, A. Hofer, E. A. Deisenhammer, M. J. Hoptman, C. Kremser, S. Golaszewski, S. Felber, W. W. Fleischhacker, and M. Delazer, "Sex differences in brain activation pattern during a visuospatial cognitive task: A functional magnetic resonance imaging study in healthy volunteers," Neurosci. Lett., vol. 344, no. 3, pp. 169-172, 2003.

[57] T. Butler, J. Imperato-McGinley, H. Pan, D. Voyer, J. Cordero, Y. S. Zhu, E. Stern, and D. Silbersweig, "Sex differences in mental rotation: Top-down versus bottom-up processing," Neuroimage, vol. 32, no. 1, pp. 445-456, 2006.

[58] G. M. Alexander, "An evolutionary perspective of sex-typed toy preferences: Pink, blue, and the brain," Archives of Sexual Behavior, vol. 32, no. 1, pp. 7-14, 2003.

[59] I. Rock, The Logic of Perception. Cambridge, MA: MIT Press, 1983.

[60] J. Bullier, J. D. Schall, and A. Morel, "Functional streams in occipito-frontal connections in the monkey," Behavioural Brain Res., vol. 76, no. 1-2, pp. 89-97, 1996.

[61] A. D. Milner, Y. Paulignan, H. C. Dijkerman, F. Michel, and M. Jeannerod, "A paradoxical improvement of misreaching in optic ataxia: New evidence for two separate neural systems for visual localization," Proc. Royal Society of London B, vol. 266, no. 1434, pp. 2225-2229, 1999.

[62] A. Paivio and J. M. Clark, "Static versus dynamic imagery," in Imagery and Cognition, C. Cornoldi and M. A. McDaniel, Eds. New York: Springer-Verlag, 1991, pp. 221-245.

[63] J. Connellan, S. Baron-Cohen, S. Wheelwright, A. Batki, and J. Ahluwalia, "Sex differences in human neonatal social perception," Infant Behavior and Develop., vol. 23, no. 1, pp. 113-118, 2000.

[64] D. J. Law, J. W. Pellegrino, and E. B. Hunt, "Comparing the tortoise and the hare: Gender differences and experience in dynamic spatial reasoning tasks," Psychol. Sci., vol. 4, no. 1, pp. 35-40, 1993.

[65] E. Poduska and D. G. Phillips, "The performance of college students on Piaget-type tasks dealing with distance, time, and speed," J. Res. Sci. Teaching, vol. 23, no. 9, pp. 845-848, 1986.
[66] J. R. Thomas and K. E. French, "Gender differences across age in motor performance: A meta-analysis," Psychol. Bull., vol. 98, no. 2, pp. 260-282, 1985.

[67] J. Hall and D. Kimura, "Sexual orientation and performance on sexually dimorphic motor tasks," Archives of Sexual Behavior, vol. 24, no. 4, pp. 395-407, 1995.

[68] N. V. Watson and D. Kimura, "Nontrivial sex differences in throwing and intercepting: Relation to psychometrically-defined spatial functions," Personality and Individual Differences, vol. 12, no. 5, pp. 375-385, 1991.

[69] W. H. Overman, B. J. Pate, K. Moore, and A. Peuster, "Ontogeny of place learning in children as measured in the radial arm maze, Morris search task, and open field task," Behavioral Neurosci., vol. 110, no. 6, pp. 1205-1228, 1996.

[70] M. H. Bornstein, "On the development of color naming in young children: Data and theory," Brain and Language, vol. 26, no. 1, pp. 72-93, 1985.

[71] D. B. Judd, "Colorblindness and the detection of camouflage," Science, vol. 97, no. 2529, pp. 544-546, 1943.

[72] J. Pokorny, V. C. Smith, G. Verriest, and A. G. L. G. Pinckers, Congenital and Acquired Color Vision Defects. New York: Grune \& Stratton, 1979.

[73] S. H. C. Hendry and R. C. Reid, "The koniocellular pathway in primate vision," Annu. Rev. Neurosci., vol. 23, pp. 127-153, 2000.

[74] N. J. Dominy and P. W. Lucas, "Ecological importance of trichromatic vision to primates," Nature, vol. 410, no. 6826, pp. 363-366, 2001.

[75] J. Nathans, "The evolution and physiology of human color vision: Insights from molecular genetic studies of visual pigments," Neuron, vol. 24 , no. 2, pp. 299-312, 1999.

[76] S. K. Shyue, D. Hewett-Emmett, H. G. Sperling, D. M. Hunt, J. K. Bowmaker, J. D. Mollon, and W. H. Li, "Adaptive evolution of color vision genes in higher primates," Science, vol. 269, no. 5228, pp. 1265-1267, 1995.

[77] M. J. Morgan, A. Adam, and J. D. Mollon, "Dichromats detect colour-camouflaged objects that are not detected by trichromats," Proc. Royal Society of London B, vol. 248, no. 1323, pp. 291-295, 1992.

[78] N. H. Hess and E. H. Hagen, "Sex differences in indirect aggression: Psychological evidence from young adults," Evolution and Human Behavior, vol. 27, no. 3, pp. 231-245, 2006.

[79] D. F. Halpern, Sex Differences in Cognitive Abilities. Hillsdale: Lawrence Erlbaum Assoc., 1986.

[80] J. McGlone, "Sex differences in human brain asymmetry: A critical survey," Behavioral Brain Sci., vol. 3, no. 2, pp. 215-263, 1980.

[81] D. Kimura, "Sex differences in the brain," Sci. Amer., vol. 267, no. 3, pp. 118-125, 1992.

[82] A. Large, J. Beheshti, and T. Rahman, "Design criteria for children's web portals: The users speak out," J. Amer. Soc. Inform. Sci. Technol., vol. 53, no. 2, pp. 79-94, 2002. 
[83] B. A. Shaywitz, S. E. Shaywitz, K. R. Pugh, R. T. Constable, P. Skudlawski, R. K. Fulbright, R. A. Bronen, J. M. Fletcher, D. P. Shankwiler, L. Katz, and J. C. Gore, "Sex differences in the functional organization of the brain for language," Nature, vol. 373, no. 6515, pp. 607-609, 1995.

[84] E. C. Bell, M. C. Wilson, A. H. Wilman, S. Dave, and P. H. Silverstone, "Males and females differ in brain activation during cognitive tasks," Neuroimage, vol. 30, no. 2, pp. 529-538, 2006.

[85] A. Dubb, R. Gur, B. Avants, and J. Gee, "Characterization of sexual dimorphism in the human corpus callosum," Neuroimage, vol. 20, no. 1, pp. 512-519, 2003.

[86] K. Pugh, B. Shaywitz, S. E. Shaywitz, R. T. Constable, P. Skudlarski, R. Fulbright, R. Bronen, D. Shankweiler, L. Katz, J. Fletcher, and J. Gore, "Cerebral organization of component processes in reading," Brain, vol. 119, no. 4, pp. 1221-1238, 1996.

[87] S. L. Rossell, E. T. Bullmore, S. C. Williams, and A. S. David, "Sex differences in functional brain activation during a lexical visual field task," Brain and Language, vol. 80, no. 1, pp. 97-105, 2002.

[88] A. Vrechopoulos, R. O’Keefe, G. Doukidis, and G. Siomkos, "Virtual store layout: An experimental comparison in the context of grocery retail," $J$. Retailing, vol. 80, no. 1, pp. 13-22, 2004.

[89] D. A. Griffith, "An examination of the influences of store layout in online retailing," J. Bus. Res., vol. 58, no. 10, pp. 1391-1396, 2005.

[90] K. Larson and M. Czerwinski, "Web page design: Implications of memory, structure and scent for information retrieval," in Proc. Conf. Human Factors in Computing Systems (CHI 1998), 1998, pp. 25-32.

[91] J. S. Ajuha and J. Webster, "Perceived disorientation: An examination of a new measure to assess web design effectiveness," Interacting with Computers, vol. 14, no. 1, pp. 15-29, 2001.

[92] T. P. Novak, D. L. Hoffman, and Y.-F. Yung, "Measuring the customer experience in online environments: A structural modeling approach," Marketing Sci., vol. 19, no. 1, pp. 22-42, 2000.

[93] J. S. Chafetz, Handbook of the Sociology of Gender. New York: Springer, 1999.

[94] K. Lucas and J. L. Sherry, "Sex differences in video game play: A communication-based explanation," Commun. Res., vol. 31, no. 5, pp. 499-523, 2004.

[95] P. Howard, L. Rainie, and S. Jones, "Days and nights on the internet: The impact of a diffusing technology," in The Internet and Everyday Life, B. Wellman and C. Haythornthwaite, Eds. Oxford: Blackwell, 2002, pp. 45-73.
Eric Stenstrom is an FQRSC research fellow (Fonds québécois de la recherche sur la société et la culture) and a Ph.D. student in Marketing at the John Molson School of Business at Concordia University, Montreal, $\mathrm{BC}$, Canada. He is currently conducting research in consumer psychology from a multidisciplinary approach under the supervision of Dr. Gad Saad. His main areas of interest include online consumer behavior, the physiological and evolutionary underpinnings of consumption, and indices of research productivity. He has presented work at the Administrative Sciences Association of Canada Annual Meetings.

Philippe Stenstrom is an NSERC research fellow (Natural Sciences and Engineering Research Council of Canada) and a Ph.D. student in Experimental Psychology at the Universite de Montréal, Montreal, QC, Canada. He is currently conducting research at the Sleep Research Center of Montreal's Sacré Coeur Hospital under the supervision of Dr. Tore A. Nielsen. His research interests include the cognitive neuroscience of sleep and evolutionary psychology. He has published in Sleep, Dreaming, Journal of Sleep Research, and Consciousness and Cognition.

Gad Saad received the Ph.D. degree from Cornell University in 1994. He is an Associate Professor of Marketing at the John Molson School of Business, Concordia University, Montreal, QC, Canada. He has held Visiting Associate Professorships at Cornell University, Dartmouth College, and the University of California-Irvine. His key research interests lie at the nexus of evolutionary theory, decision-making, and consumer behavior. He recently published the first scholarly book on the links between Darwinian theory and consumption titled The Evolutionary Bases of Consumption. His interdisciplinary interests are exemplified by the diversity of his publication outlets, which include journals in business, psychology, economics, medicine, and bibliometrics. He was the recipient of the faculty-wide Distinguished Teaching Award in 2000, and was listed as one of the "hot professors" of Concordia University in the 2001 and 2002 Maclean's reports on Canadian universities.

Soumaya Cheikhrouhou is working toward the Ph.D. degree in Administration (Marketing) at the John Molson School of Business, Concordia University, Montreal, QC, Canada, and is an instructor at HEC Montréal. Her research interests focus on multimodal sensory perception, perceptual biases, and consumer judgment. She is also interested in services marketing and electronic marketing. She has published papers in this area in the Canadian Journal of Administrative Sciences and in the Administrative Sciences Association of Canada Conference Proceedings. 